\title{
Relativity in the IERS Conventions
}

\author{
Gérard Petit ${ }^{1}$ \\ ${ }^{1}$ Bureau International des Poids et Mesures \\ 92312 Sèvres Cedex, France \\ email: gpetit@bipm.org
}

\begin{abstract}
In the last years, a fully general relativistic definition of reference systems and of their application to astronomy and geodesy has been passed into Resolutions of the scientific unions, following work of several working groups and of the community at large. In this community, the role of the International Earth Rotation and Reference systems Service (IERS) is to generate the terrestrial and celestial reference systems and the transformation between them, and the IERS Conventions provide the set of models and procedures used in the generation of IERS products. It is therefore essential that the IAU framework for relativity is introduced in the IERS Conventions, and that this is done consistently and completely throughout the document. The paper reviews relativistic aspects in the IERS Conventions and presents recent and on-going work aiming at providing a complete and consistent presentation for a new reference edition of the IERS Conventions, expected to appear in the next year.
\end{abstract}

Keywords. Reference systems, relativity, time

The International Earth Rotation and Reference systems Service (IERS) has been established in 1988 by the International Astronomical Union (IAU) and the International Union of Geodesy and Geophysics (IUGG). Its primary objectives are to serve the astronomical, geodetic and geophysical communities by providing realizations of the celestial (ICRF) and terrestrial (ITRF) reference systems and parameters allowing to transform between the two systems. In addition the IERS provides geophysical data to interpret time/space variations in the ICRF, ITRF or earth orientation parameters, and to model such variations. The IERS Conventions present the set of standards, constants, models and procedures to generate the above-mentioned products. They are formalized in successive reference versions, the IERS Standards (1992) (McCarthy, 1992), the IERS Conventions (1996) (McCarthy, 1996), and IERS Conventions (2003) (McCarthy \& Petit, 2004).

We recall the relativistic framework built up by the set of IAU Resolutions in Section 1 and present in Section 2 the work in progress or already realized concerning all relativistic aspects in the IERS Conventions.

\section{The relativistic framework}

In order to describe observations in astronomy and geodesy, one has to choose the relativistic reference systems best suited to the problem at hand. A barycentric celestial reference system (BCRS) should be used for all experiments not confined to the vicinity of the Earth, while a geocentric celestial reference system (GCRS) is physically adequate to describe processes occurring in the vicinity of the Earth. These systems have been defined in a series of Resolutions passed by scientific Unions, mostly the IAU, in the past 20 years, see a more complete description of the work until year 2000 in (Soffel et al., 2003). 
1991 and the following years. The reference systems were first defined by the IAU Resolution A4 (1991) which contains nine recommendations, the first four of which are summarized below.

In the first recommendation, the metric tensor for space-time coordinate systems $(t, \mathbf{x})$ centered at the barycenter of an ensemble of masses is recommended in the form

$$
\begin{aligned}
& g_{00}=-1+2 U(t, \mathbf{x}) / c^{2}+\mathcal{O}\left(c^{-4}\right), \\
& g_{0 i}=\mathcal{O}\left(c^{-3}\right), \\
& g_{i j}=\delta_{i j}\left(1+2 U(t, \mathbf{x}) / c^{2}\right)+\mathcal{O}\left(c^{-4}\right),
\end{aligned}
$$

where $c$ is the speed of light in vacuum $(c=299792458 \mathrm{~m} / \mathrm{s})$ and $U$ is the Newtonian gravitational potential (here a sum of the gravitational potentials of the ensemble of masses, and of a external potential generated by bodies external to the ensemble, the latter potential vanishing at the origin). The recommended form of the metric tensor can be used, not only to describe the barycentric reference system of the whole solar system, but also to define the geocentric reference system centered in the center of mass of the Earth with $U$, now depending upon geocentric coordinates.

In the second recommendation, the origin and orientation of the spatial coordinate grids for the barycentric and geocentric reference systems are defined.

The third recommendation defines TCB (Barycentric Coordinate Time) and TCG (Geocentric Coordinate Time) as the time coordinates of the BCRS and GCRS, respectively, and, in the fourth recommendation, another time coordinate named TT (Terrestrial Time), is defined for the GCRS as

$$
\mathrm{TT}=\mathrm{TCG}-\mathrm{L}_{\mathrm{G}} \times\left(\mathrm{JD}_{\mathrm{TCG}}-T_{0}\right) \times 86400,
$$

where $\mathrm{JD}_{\mathrm{TCG}}$ is the TCG Julian date, $T_{0}=2443144.5003725$ and where $L_{G}=U_{G} / c^{2}$ with $U_{G}$ being the gravity potential on the geoid.

Note that the IUGG, in its Resolution 2 (1991), endorsed the IAU Recommendations and explicitly based its definition of Terrestrial Reference Systems on the IAU relativistic framework.

In 1997 the IAU has supplemented the framework by one more recommendation stating that no scaling of spatial axes should be applied in any reference system, even if scaled time coordinate like TT is used for convenience of an analysis (this is in relation with e.g. discussions on the VLBI model, see next Section).

2000 and the recent years. In the years following the adoption of the IAU'1991 Resolution, it became obvious that this set of recommendations was not sufficient, especially with respect to planned astrometric missions with $\mu$ as-accuracies and with respect to the expected improvement of atomic clocks and the planned space missions involving such clocks and improved time transfer techniques. For that reason the IAU WG "Relativity for celestial mechanics and astronomy" together with the BIPM-IAU Joint Committee for relativity suggested an extended set of Resolutions that was finally adopted at the IAU General Assembly in Manchester in the year 2000 as Resolutions B1.3 to B1.5 and B1.9.

Resolution B1.3 concerns the definition of Barycentric Celestial Reference System (BCRS) and Geocentric Celestial Reference System (GCRS). The Resolution recommends to write the metric tensor of the BCRS in the form

$$
\begin{aligned}
g_{00} & =-1+2 w / c^{2}-2 w^{2} / c^{4}+\mathcal{O}\left(c^{-5}\right), \\
g_{0 i} & =-4 / c^{3} w^{i}+\mathcal{O}\left(c^{-5}\right), \\
g_{i j} & =\delta_{i j}\left(1+2 w / c^{2}\right)+\mathcal{O}\left(c^{-4}\right) .
\end{aligned}
$$


where $w$ is a scalar potential and $w^{i}$ a vector potential. This extends the form of the metric tensor given in (1.1), so that its accuracy is now sufficient for all applications foreseen in the next years, including those involving accurate space clocks. For the GCRS, Resolution B1.3 also adds that the spatial coordinates are kinematically non-rotating with respect to the barycentric ones.

Resolution B1.4 provides the form of the expansion of the post-Newtonian potential of the Earth to be used with the metric of Resolution B1.3.

Resolution B1.5 applies the formalism of Resolutions B1.3 and B1.4 to the problems of time transformations and realization of coordinate times in the solar system. Resolution B1.5 is based upon a mass monopole spin dipole model. It provides an uncertainty not larger than $5 \times 10^{-18}$ in rate and, for quasi-periodic terms, not larger than $5 \times 10^{-18}$ in rate amplitude and $0.2 \mathrm{ps}$ in phase amplitude, for locations farther than a few solar radii from the Sun. The same uncertainty also applies to the transformation between TCB and TCG for locations within $50000 \mathrm{~km}$ of the Earth.

Some shortcomings appeared in the definition of TT (1.2) when considering accuracy below $10^{-17}$ : the uncertainty in the determination of $U_{G}$ is limited, the surface of the geoid is difficult to realize so that it is difficult to determine the potential difference between the geoid and the location of a clock, and in addition the geoid varies with time. Therefore it was decided to desociate the definition of TT from the geoid while maintaining continuity with the previous definition. The constant $L_{G}$ was turned into a defining constant with its value fixed to $6.969290134 \times 10^{-10}$ in Resolution B1.9, which therefore removes the limitations mentioned above when realizing TT from clocks onboard terrestrial satellites.

Finally in 2006 it was decided to redefine the coordinate time TDB, which had been introduced by the IAU in 1976 as a dynamical time scale for barycentric ephemerides. As it had not been unambiguously defined, multiple realizations of TDB were possible. Because such realizations are still widely used for barycentric ephemerides, IAU Resolution B3 (2006) was passed to define TDB as the following linear transformation of TCB:

$$
\mathrm{TDB}=\mathrm{TCB}-L_{B} \times\left(\mathrm{JD}_{\mathrm{TCB}}-T_{0}\right) \times 86400+T D B_{0},
$$

where $\mathrm{JD}_{\mathrm{TCB}}$ is the TCB Julian date and where $L_{B}=1.550519768 \times 10^{-8}$ and $T D B_{0}=$ $-6.55 \times 10^{-5} s$ are defining constants. Figure 1 shows graphically the relationships between the time scales following the IAU Resolutions of 1991, 2000 and 2006.

\section{Relativistic aspects in the IERS Conventions}

In the work to update the IERS Conventions (2003), relativistic aspects cover three topics. The first one is to review the nomenclature used throughout the document in order to ensure its consistency, both internally and with the Unions' recommendations. The second one concerns chapter 10 (models for space-time coordinates and equations of motion) where, in a recent update (see http://tai.bipm.org/iers/convupdt/convupdt.html) the transformation from proper time to coordinate time in the vicinity of the Earth is treated and numerical examples are provided for the different terms in the relativistic expression for the acceleration of an Earth satellite. The third topic concerns chapter 11 (models for signal propagation) and covers models for VLBI and (radio and laser) ranging techniques.

Nomenclature. Nomenclature issues in the IERS Conventions can be loosely classified in two categories, although several issues are interconnected. The first type, and the most important for what concerns relativistic aspects, is about the designation of coordinates and coordinate quantities; the second type relates to the definition and realization of 
reference systems and to the transformation between celestial and terrestrial reference systems.

In the first category, the wording used to designate coordinates and coordinate quantities (e.g. space coordinates, gravitational constants $G M$, etc...) has to be reviewed. The IAU Commission "Relativity in fundamental astronomy" (RIFA) has proposed a conventional wording (Klioner, 2008) that can be summarized by two main rules:

- All quantities intended for use with time scale XX (e.g. TDB) should be called "XX-compatible quantities" and the corresponding values "XX-compatible values". In the case of constants having the same value in BCRS and GCRS (e.g. mass parameters $\mu=G M$ of celestial bodies) the value can be called "unscaled".

- Avoid attaching any adjectives to the names of the units second and meter for numerical values of these quantities. For example, the expression "The interval is $\mathrm{xx}$ seconds of TDB" should be written as "The TDB interval is xx seconds".

In the Conventions (2003) a variety of wordings are used, which can be classified into three types:

- One makes use of the word "unit", like in "[so-called] TDB unit" (for cases when a scaled coordinate time is used, here TDB), or in "TCB (SI) units" TCB (SI) units (for cases when an unscaled coordinate time is used).

- One uses the word "scale", like in "ITRF ... uses the TT scale".

- One uses a full set of words in a sentence, like in "... coordinates consistent with TDB".

The first two types may be corrected following the above-mentioned rules, while expressions of the third type are acceptable in the proposed nomenclature.

The second category of nomenclature issues mostly concerns Chapter 5 (transformation between celestial and terrestrial systems) and Chapter 4 (Terrestrial Reference System). Both chapters have been revised (see http://tai.bipm.org/iers/convupdt/convupdt.html) following the work of the IAU Division I Working Group "Nomenclature for Fundamental Astronomy" (NFA), see http://syrte.obspm.fr/iauWGnfa/NFA_Glossary.html and (Capitaine, 2008).

Chapter 10: Models for space-time coordinates and the equations of motion. This chapter has been updated in 2008 and the presentation of coordinate time scales now accounts for all IAU Resolutions (see section 1). The relationship between all time scales used in this context is shown on Figure 1, taken from this chapter in the IERS Conventions.

In addition, a new section covers the transformation between proper time and coordinate time in the vicinity of the Earth (typically up to geosynchronous orbit or slightly above). Evaluating the contributions of the higher order terms in the metric (1.3) applied to the geocentric reference system GCRS, it is found that the IAU'1991 metric (1.1) is sufficient for time and frequency applications in the GCRS in the light of present clock accuracies.

When considering TT as coordinate time, the proper time of a clock $A$ located at the GCRS coordinate position $\mathbf{x}_{A}(t)$, and moving with the coordinate velocity $\mathbf{v}_{A}$, is

$$
\frac{d \tau_{A}}{d \mathrm{TT}}=1+L_{G}-1 / c^{2}\left[\mathbf{v}_{A}^{2} / 2+U_{\mathrm{E}}\left(\mathbf{x}_{A}\right)+U_{\text {ext }}\left(X_{\mathrm{A}}\right)-U_{\text {ext }}\left(X_{\mathrm{E}}\right)-x_{A}^{i} \partial_{i} U_{\text {ext }}\left(X_{\mathrm{E}}\right)\right]
$$

Here, $U_{\mathrm{E}}$ denotes the Newtonian potential of the Earth at the position $\mathbf{x}_{A}$ of the clock in the geocentric frame, and $U_{\text {ext }}$ is the sum of the Newtonian potentials of the other bodies (mainly the Sun and the Moon) computed at a location $X$ in barycentric coordinates, either at the position $X_{\mathrm{E}}$ of the Earth center of mass, or at the clock location $X_{\mathrm{A}}$. The 


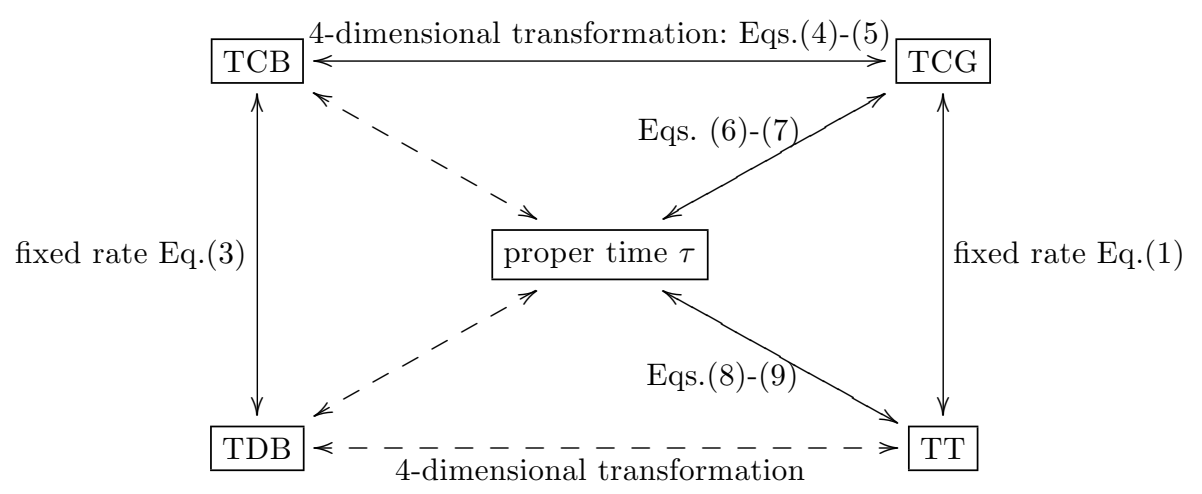

Figure 1. Various relativistic time scales and their relations. Each of the coordinate time scales TCB, TCG, TT and TDB can be related to the proper time $\tau$ of an observer, provided that the trajectory of the observer in the BCRS and/or GCRS is known. See chapter 10 in the IERS Conventions web site at http://tai.bipm.org/iers/convupdt/convupdt.html for reference to the transformations listed in the Figure.

last three terms are tidal terms and their contribution will be limited to below $1 \times 10^{-15}$ in frequency and a few ps in time amplitude up the GPS orbit, so they may be skipped depending on the uncertainty required. Nevertheless, some care needs to be taken when evaluating the Earth's potential $U_{\mathrm{E}}$ at the location of the clock as the uncertainty in $U_{\mathrm{E}}$ should be consistent with the uncertainty expected on (2.1). Analytical formulas may be specified e.g. for GPS (Ashby, 2003; Kouba, 2004), however a numerical integration of equation (2.1) using the proper development for the potential is always worth using. This is specially the case for low Earth orbit satellites (see e.g. Larson et al., 2007), where analytical expressions may be significantly in error or even completely misleading.

Chapter 11: Models for signal propagation. In the Conventions (2003), chapter 11 describes the relativistic model for VLBI time delay and for Laser ranging.

In the short term, no change is expected in the VLBI model which provides an accuracy below 1 ps, but it should be reviewed in the next years in view of the planned improvement in VLBI observations (Behrend et al., 2008). However some cosmetic changes may be introduced, linked to the nomenclature. In order to describe the possible effect of an incorrect use of coordinates and coordinate quantities, it is worth reminding the past history of this model since its introduction in 1990. In 1990, the so-called "Consensus model" was adopted at a USNO workshop. This was in an era before the adoption of the IAU'1991 Resolution so that, although relativity was carefully accounted for, the currently agreed notations did not exist at that time. The model appeared first in the IERS Standards (1992), but was modified in the IERS Conventions (1996), erroneously intending to comply with the IAU/IUGG'1991 Resolutions, on the basis that "as the time argument is now based on TAI ..., distance estimates from these conventions will now be consistent 'in principle' with physical distances". However, the change so introduced in the Conventions (1996) would have produced space coordinates which would have differed from the usual TT-compatible space coordinates by a scale change of $1.4 \times 10^{-9}$. Furthermore it must be recognized that the goal stated in the Conventions (1996) is not achievable as no coordinate quantity can be consistent with a physical (proper) quantity over the extension of the Earth. The change was however never implemented by analysis centers and the model was eventually restored in its original form in the IERS Conventions (2003) with additional explanations. Indeed, the consensus model can provide either TT-compatible space coordinates when used with raw VLBI 
(TT-compatible) delays (as is the usual case in VLBI analysis), or it could provide TCGcompatible space coordinates if used with delays transformed to be TCG-compatible. Such issues, which may result in scale differences in case of misinterpretation, are reexamined whenever the scale of the terrestrial reference frame is discussed, however it should be stressed that no ambiguity exists in the present model of the Conventions.

Finally, the section on laser ranging is to be re-examined in order to cover all ranging techniques by electromagnetic signals in the vicinity of the Earth (up to the Moon). As it has been shown (Klioner, 2007) that post-post Newtonian terms are not required in view of the present uncertainty, no significant model change is expected.

\section{Conclusions}

The relativistic framework specified by IAU Resolutions in 1991 and 2000, and supplemented by additional Recommendations, is now complete and adapted to the current and planned applications in astrometry and space geodesy. Work remains to be done to apply it in all fields and, in some cases, a conventionally adopted nomenclature is still not widely used. This work is under way in IAU working groups (NSFA) and commissions (RIFA) and in the IERS Conventions center. It should be put into application in the next reference edition of the IERS Conventions expected in the near future.

\section{References}

Ashby, N. 2003, Living Rev. Relativity, 6, 1, http://www.livingreviews.org/lrr-2003-1

Behrend D. et al. 2008, IAG Symposia series (Springer Verlag), 133, Part 5, 833

Capitaine, N. 2008, Proc. Journées SRST, Lohrmann-Observatorium and Observatoire de Paris, 46

Klioner, S. A. 2007, IERS Workshop on Conventions, http://www.bipm.org/en/events/iers

Klioner, S. A. 2008, AESA, 478-3, 951

Kouba, J. 2004, GPS Solutions, 8,3, 170

Larson, K. M., Ahsby, N., Hackman, C., \& Bertiger, W. 2007, Metrologia, 44, 484

McCarthy, D. D. (ed.) 1992, IERS TN13, Observatoire de Paris, 150 p.

McCarthy, D. D. (ed.) 1996, IERS TN21, Observatoire de Paris, 95 p.

McCarthy, D. D. \& Petit, G. (eds.) 2004, IERS TN32, Verlag des BKG, 127 p.

Soffel, M., Klioner, S., Petit, G., Wolf, P., et al. 2003, AJ, 126(6), 2687 